\title{
Estudo infodemiológico da associação da pandemia da COVID-19 no Brasil com o volume de pesquisa na internet
}

\author{
Infodemiological study of the COVID-19 pandemic association in Brazil and the volume of internet \\ search
}

Estudio infodemiológico de la asociación de la pandemia COVID-19 en Brasil con el volumen de búsquedas en Internet

\section{Resumo}

Verificar a existência de relação entre a busca por informação na Internet sobre COVID-19 e os números de casos e óbitos pela doença no território brasileiro. Realizamos um estudo infodemiológico usando a palavra de busca "Coronavírus" no Google Trends ${ }^{\mathrm{TM}}$, para cada unidade federativa e o Distrito Federal, referentes à população, ao número de casos confirmados e ao número de óbitos. Em seguida, comparamos cada Volume de Pesquisa Relativo ao número de novos casos e óbitos por COVID-19 divulgados pelo Ministério da Saúde do Brasil durante o período de maio a junho de 2020. Os primeiros casos de COVID-19 notificados no país foram acompanhados do aumento de buscas na Internet sobre o tema, havendo uma redução do interesse ao longo do período estudado. A média de Volume de Pesquisa Relativo foi 37,8. Houve uma correlação positiva entre casos e óbitos, entretanto a correlação entre esses parâmetros e o Volume de Pesquisa Relativo comportou-se com significância negativa. Dados infodemiológicos têm permitido monitorar em tempo real surtos de doenças infecciosas em nível local e global. O Brasil apresentou comportamento inverso aos estudos anteriores. Evidenciamos uma forte relação negativa entre o acesso a conteúdo na Internet e o número de casos confirmados e óbitos por COVID-19 em todo o território brasileiro.

Palavras-chave: SARS-CoV-2; Comportamento; Internet; Incidência; Mortalidade.

\begin{abstract}
To verify the existence of a relationship between the search for information on the Internet about COVID-19 and the number of cases and deaths caused by the disease in the Brazilian territory. We performed an infodemiological study using the search word "Coronavirus" on Google Trends ${ }^{\mathrm{TM}}$, for each federative unit and the Federal District, referring to the population, the number of confirmed cases and the number of deaths. We then compared each Research Volume Relating to the number of new cases and deaths from COVID-19 reported by the Ministry of Health of Brazil during the period from May to June 2020. The first cases of COVID-19 reported in the country were accompanied by an increase in Internet searches on the subject, with a reduction in interest over the period studied. The average Relative Research Volume was 37.8. There was a positive correlation between cases and deaths, however the correlation between these parameters and the Relative Research Volume behaved with negative significance. Infodemiological data have allowed real-time monitoring of outbreaks of infectious diseases at local and global levels. Brazil showed the opposite
\end{abstract}


behavior to previous studies. We evidenced a strong negative relationship between access to content on the Internet and the number of confirmed cases and deaths from COVID-19 throughout the Brazilian territory.

Keywords: SARS-CoV-2; Behavior; Internet; Incidence; Mortality.

\begin{abstract}
Resumen
Verificar la existencia de una relación entre la búsqueda de información en Internet sobre COVID-19 y el número de casos y muertes causadas por la enfermedad en el territorio brasileño. Realizamos un estudio infodemiológico utilizando la palabra de búsqueda "Coronavirus" en Google Trends TM, para cada unidad federativa y Distrito Federal, haciendo referencia a la población, el número de casos confirmados y el número de defunciones. Luego comparamos cada Volumen de Investigación Relativo al número de nuevos casos y muertes por COVID-19 reportados por el Ministerio de Salud de Brasil durante el período de mayo a junio de 2020. Los primeros casos de COVID-19 reportados en el país fueron seguidos por el aumento de las búsquedas en Internet sobre el tema, con una reducción del interés durante el período estudiado. El promedio del volumen de investigación relativo fue de 37,8. Hubo una correlación positiva entre casos y muertes, sin embargo, la correlación entre estos parámetros y el Volumen Relativo de Investigación se comportó con significancia negativa. Los datos infodemiológicos han permitido el seguimiento en tiempo real de los brotes de enfermedades infecciosas a nivel local y mundial. Brasil mostró una conducta contraria a estudios anteriores. Evidenciamos una fuerte relación negativa entre el acceso a contenidos en Internet y el número de casos confirmados y muertes por COVID-19 en todo el territorio brasileño.
\end{abstract}

Palabras clave: SARS-CoV-2; Conducta; Internet; Incidencia; Mortalidad.

\title{
1. Introdução
}

Em dezembro de 2019, a China passou a registrar e a atender vários casos de pneumonia de etiologia desconhecida e com elevada virulência e mortalidade. Após análises moleculares, foi identificado que se tratava de um novo coronavírus, o Sars-CoV-2. Em 11 de fevereiro de 2020, a Organização Mundial da Saúde (OMS) nomeou oficialmente a doença de coronavirus disease 2019 (COVID-19). O número crescente de casos em todo o mundo levou a OMS a declarar o surto de coronavírus como uma pandemia em 11 de março de 2020, e desde a sua descoberta continua sendo disseminado em larga escala (Huang, Wang, Li, Ren, Zhao, Huet al., 2020; Chen, Zhou, Dong, Qu, Gong, Han, et al., 2020; Zhu, Zhang, Wang, Li, Yang, Song, et al., 2020; Wang, Pan, Zhang, Fan, Han, Zhao et al., 2020; Ferreira, Almeida, Mattos \& Oliveira, 2020; OMS, 2021).

A identificação do novo coronavírus fez com que usuários de todo o mundo fizessem buscas na Internet a fim de adquirir informações sobre a COVID-19. O auge das buscas foi registrado em janeiro de 2020, mas, com o reconhecimento de que se tratava de uma pandemia, houve um aumento do interesse público em 12 de março de 2020 (Effenberger, Kronbichler, Shin, Mayer, Tilg \& Perco, 2020).

A população brasileira passa em média seis horas por dia conectada à Internet; utilizando-a de diversas formas, entre elas: buscar informações sobre saúde. A análise de como as pessoas acessam, pesquisam, comunicam e compartilham informações obtidas na Internet pode fornecer informações sobre o comportamento das populações relacionado à saúde que antes eram indisponíveis (Moretti, Oliveira \& Silva, 2012; Mavragani, Ochoa \& Tsagarakis, 2018; Hosseini, Asemi \& Shabani, 2019).

O uso metodológico de informática para analisar o comportamento na Internet aplicado à saúde pública é chamado de infodemiologia, um método de monitoramento de consultas que cresce significativamente por permitir que dados disponíveis em formato eletrônico possam ser coletados e analisados por métodos estatísticos, gerando dados para tomar as medidas necessárias para minimizar a propagação de uma doença (Eysenbach, 2009; Tana, 2019).

Considerando o elevado número de casos e óbitos por COVID-19 no Brasil e a escassez de dados infodemiológicos específicos para o cenário brasileiro, este artigo objetivou analisar a relação entre o comportamento coletivo por busca de informações sobre coronavírus na Internet e os números de casos e mortes disponibilizados pelo Ministério da Saúde brasileiro. 


\section{Metodologia}

Trata-se de um estudo de infodemiologia longitudinal, retrospectivo, passivo, bruto e baseado na demanda. Segundo Eysenbach (2009), esse método é realizado por meio da coleta de dados provenientes de mecanismos de busca em ferramentas da Internet que caracterizam o comportamento coletivo por busca de informações. Trata-se de um método passivo por analisar as tendências na Internet de forma automática, sem envolver ativamente os usuários. Além disso, é um método bruto por ser realizado usando um termo de pesquisa em um mecanismo de busca, que fornece o número de ocorrências ao longo do tempo.

A coleta de dados foi realizada por dois pesquisadores independentes e que acessaram os sites do Instituto Brasileiro de Geografia e Estatística (IBGE) (https://www.ibge.gov.br/institucional/o-ibge.html), do Departamento de Informática do Sistema Único de Saúde (Datasus) (https://datasus.saude.gov.br/) e o site do Google Trends (GT) (https://trends.google.com.br/) a partir das suas residências. Antes da pesquisa, todos os cookies existentes foram excluídos do navegador. Quaisquer desacordos foram resolvidos por consenso antes da análise final.

O IBGE é o principal provedor de dados e informações do Brasil, oferecendo informações estatísticas dos mais diversos segmentos da sociedade. O IBGE (2021) informa que o Brasil possui uma área territorial de 8,51 milhões de km² , o que equivale a quase $50 \%$ do território da América do Sul, sendo a quinta maior área territorial do planeta. Ainda, possui uma população estimada em 211.755.692 milhões de habitantes, sendo o país com a sexta maior população, com densidade demográfica de 24,47 habitantes por $\mathrm{km}^{2}$, distribuída nas 27 unidades federativas (UFs), chamadas de estados, e o Distrito Federal (DF), sede do governo brasileiro. A representação gráfica das regiões e estados brasileiros podem ser visualizados na figura 1.

O Datasus provê dados de saúde necessários ao processo de planejamento, operação e controle. Os dados sobre casos confirmados e óbitos de COVID-19, recuperados em 5 de março, eram relativos ao período de 01 de abril até 30 de junho de 2020, para cada UF brasileira e o DF. Foram coletados dados referentes à população, ao número de casos confirmados e ao número de óbitos.

O GT é uma ferramenta on-line e gratuita que fornece em tempo real acesso a uma amostra de pesquisas reais feitas sobre um assunto específico. Essa ferramenta foi usada para recuperar dados das atividades de pesquisa de usuários da Internet sobre a COVID-19. 
Figura 1 - Mapa do Brasil mostrando as 27 Unidades Federativas, e o Distrito Federal, Brasília.

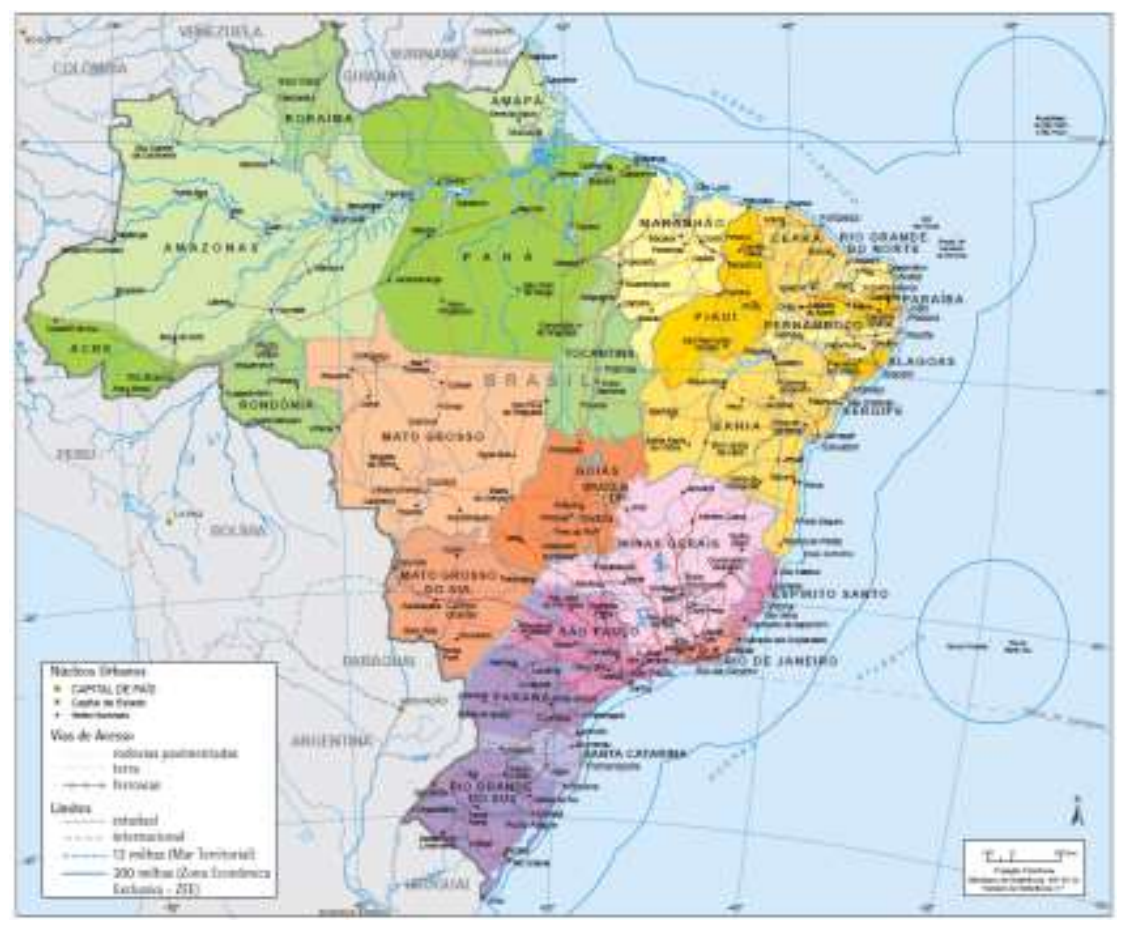

*As tonalidades de cores representam as regiões brasileiras. Verde: Região Norte, composta por sete UFs. Laranja: Região Centro-oeste, composta por três UFs e o DF. Amarelo: Região Nordeste, composta por nove UFs. Rosa: Região Sudeste, composta por quatro UFs. Lilás: Região Sul, composta por três UFs. Fonte: IBGE (2021).

Para realizar consultas on-line é essencial a adequada seleção das palavras de busca para obter resultados válidos. Inicialmente, foi feita uma busca comparativa dos resultados da tendência de busca entre as palavras "Coronavírus" e "COVID19” no GT, para verificar qual era a mais utilizada no Brasil para buscar informações. A partir daí, confirmou-se que a mais adequada para obter dados consistentes no contexto brasileiro era "Coronavírus".

Os índices do GT no Brasil foram recuperados de 01 de abril 2020 até 30 de junho de 2020. A coleta de dados ocorreu no mecanismo de busca do GT, usando como parâmetros a palavra de busca "Coronavírus", selecionando-se o país "Brasil", definindo o período como "nos últimos 90 dias" e a categoria como "Todas as categorias".

Os dados do GT são apresentados como um índice do interesse relativo da pesquisa sobre determinado tópico. Os números resultantes são dimensionados em um intervalo de 0 a 100. Os termos de pesquisa com volume baixo aparecem como "0" e o interesse máximo aparece como “100”. Para os dados recuperados do GT, utilizaremos o termo Relative Search Volume (RSV) com tradução livre para o idioma português como Volume de Pesquisa Relativa.

Essencialmente, o RSV representa a popularidade da pesquisa em um momento específico. Uma aceleração repentina do interesse da pesquisa em um tópico, em comparação com o volume de pesquisa usual, gera um pico no gráfico, que geralmente reflete o interesse de busca sobre algo que está acontecendo no mundo real. Foram coletados os RSVs de todas as UFs brasileiras e do DF.

Em relação à análise e apresentação dos dados, a entrada dos dados do Datasus e os índices diários do GT foram realizadas no programa Microsoft Excel. A análise estatística foi realizada a partir do pacote estatístico Statistical Package for the Social Sciences (SPSS), sendo calculadas as correlações de intervalo de tempo entre os picos de busca no GT, os RSVs e novos casos e óbitos. 
Inicialmente, foi realizado o teste de Kolmogorov-Smirnov para estudo da normalidade dos dados. Após a aplicação do teste e a constatação da normalidade, foi utilizado o coeficiente de correlação de Pearson, servindo como medida de correlação. O valor-p menor ou igual a 0,05 foi considerado estatisticamente significativo.

A infodemiologia deve exercer comportamento ético sobre as informações de acesso aberto de indivíduos. Dessa forma, no presente estudo, adotou-se o método passivo, analisando tendências de consulta na Internet de forma automática, sem envolver ativamente os usuários. Os dados disponíveis no IBGE e no Datasus são de domínio público. O GT, por sua vez, fornece acesso a uma amostra das solicitações de pesquisa reais feitas ao Google sem identificar os usuários, garantindo o anonimato.

\section{Resultados}

O Brasil seguiu a tendência mundial de aumentar o número de buscas sobre coronavírus. O primeiro pico de RSV sobre o tema, no Brasil, ocorreu em abril de 2020, havendo uma redução do RSV ao longo do período estudado, como pode ser visualizado no Gráfico 1.

Gráfico 1 - Volume de Pesquisa Relativa no Brasil sobre "Coronavírus” no período abr-junho/2020, Brasil.

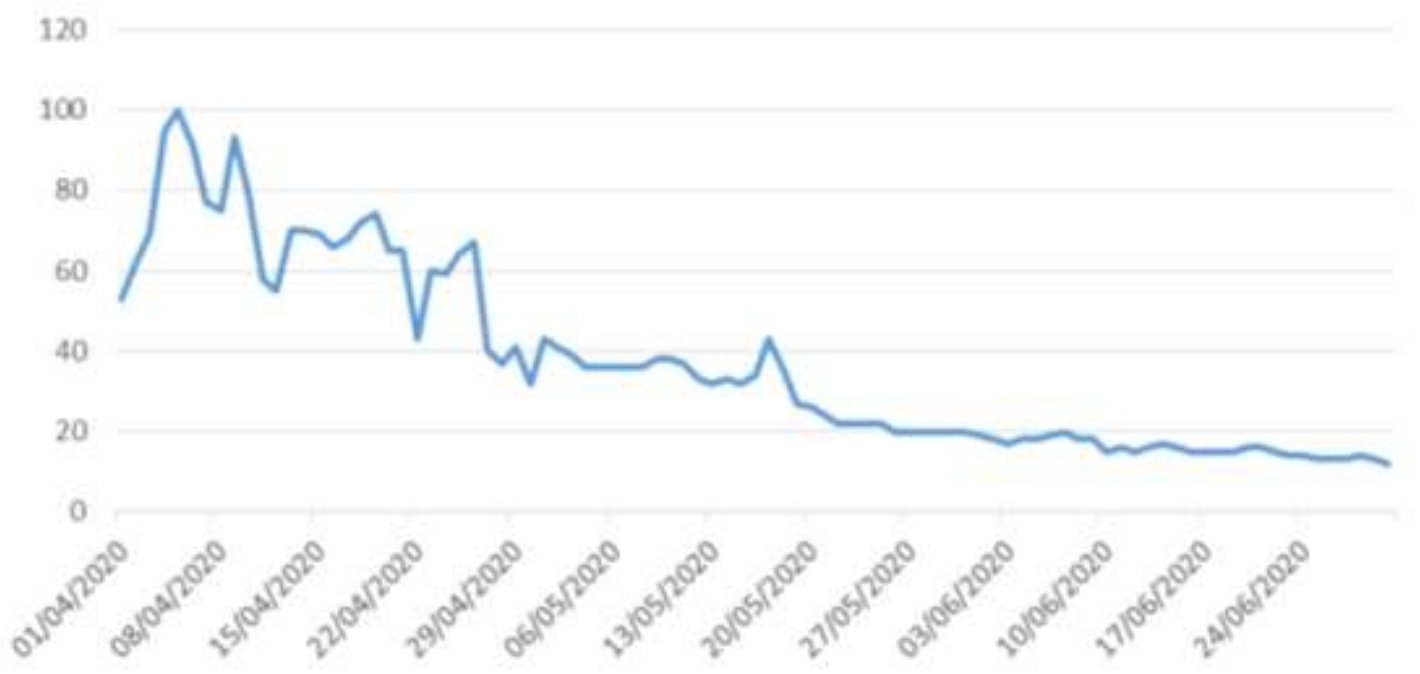

Fonte: Autores.

Considerando que o RSV varia de 0 a 100, a média brasileira atingiu o valor de 37,8. O RSV mostrou-se maior em Rondônia, mostrando um volume de pesquisa de 44, ao passo que o menor índice de busca ocorreu no Amazonas, com volume de pesquisa de 28,3. Ressalta-se que ambas as UFs se encontram na mesma região geográfica, Norte do Brasil, mas apresentaram comportamentos distintos, como pode ser visualizado no Gráfico 2. 
Gráfico 2 - Média do Volume de Pesquisa Relativa dos estados brasileiros sobre “Coronavírus” no período abr-junho/2020,

Brasil.

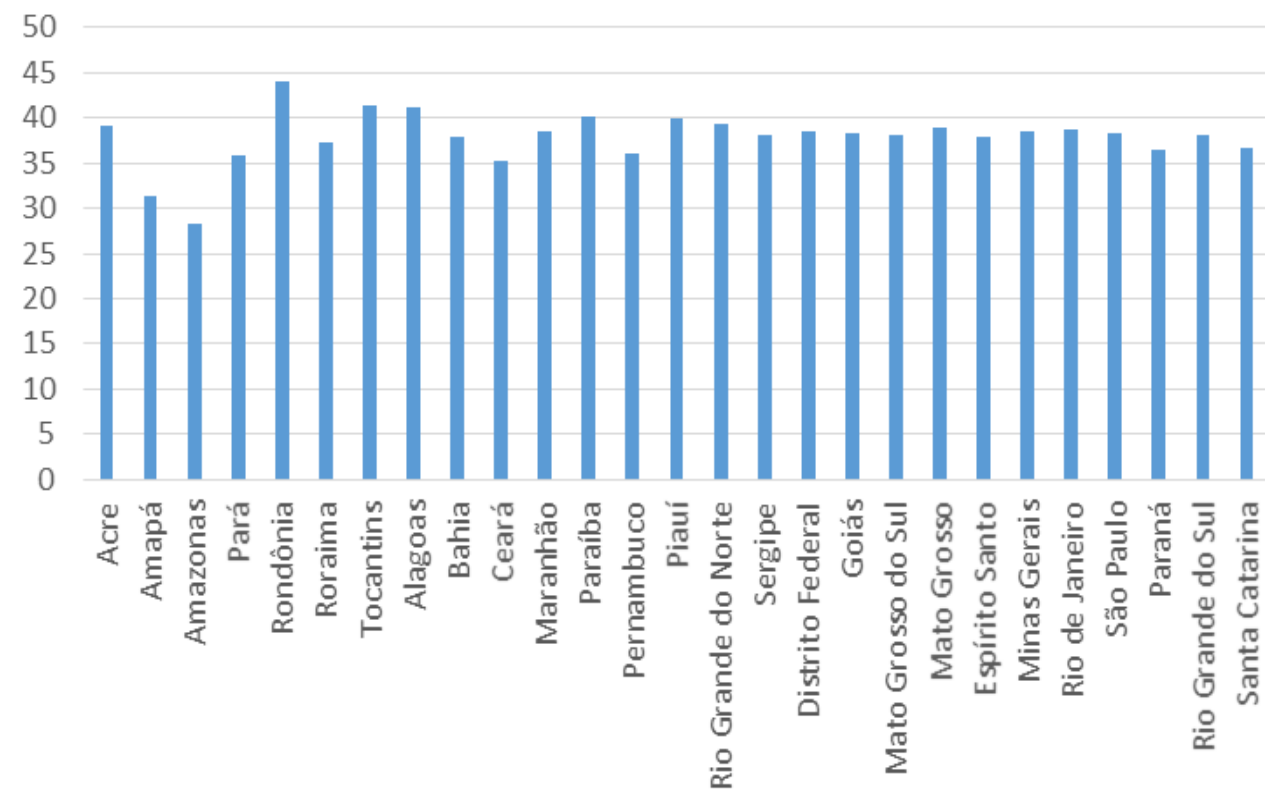

Fonte: Autores.

As correlações de Pearson testaram a relação entre o RSV da doença e a prevalência ou incidência da doença em determinado período de tempo para o coronavírus. No geral, a relação entre as variáveis estudadas foi significativa entre todos os parâmetros.

De abril a junho de 2020, observou-se uma correlação positiva entre os parâmetros epidemiológicos, casos e óbitos. De forma contrária, a correlação entre esses parâmetros e o RSV comportou-se negativamente, com significância p<0,0001, como mostra a Tabela 1 .

Tabela 1 - Coeficientes de Correlação de Pearson entre casos, óbitos e RSV no período abr-junho/2020, Brasil.

\begin{tabular}{|c|c|c|c|c|c|c|}
\hline & & Casos Novos & $\begin{array}{c}\text { Casos } \\
\text { Acumulados }\end{array}$ & $\begin{array}{l}\text { Óbitos } \\
\text { Novos }\end{array}$ & $\begin{array}{c}\text { Óbitos } \\
\text { Acumulados }\end{array}$ & Trend \\
\hline \multirow{3}{*}{$\begin{array}{l}\text { Casos } \\
\text { Novos }\end{array}$} & $\begin{array}{l}\text { Pearson } \\
\text { Correlation }\end{array}$ & 1 & $0,780^{* *}$ & $0,732^{* *}$ & $0,734^{* *}$ & $-0,386^{* *}$ \\
\hline & Sig. (2-tailed) & & 0,000 & 0,000 &, 0000 & 0,000 \\
\hline & $\mathrm{N}$ & 2457 & 2457 & 2457 & 2457 & 2457 \\
\hline \multirow{3}{*}{$\begin{array}{l}\text { Casos } \\
\text { Acumulados }\end{array}$} & $\begin{array}{l}\text { Pearson } \\
\text { Correlation }\end{array}$ & $0,780^{* *}$ & 1 & $0,696^{* *}$ & $0,942^{* *}$ & $-0,441^{* *}$ \\
\hline & Sig. (2-tailed) & 0,000 & & 0,000 & 0,000 & 0,000 \\
\hline & $\mathrm{N}$ & 2457 & 2457 & 2457 & 2457 & 2457 \\
\hline \multirow{3}{*}{ Óbitos Novos } & $\begin{array}{l}\text { Pearson } \\
\text { Correlation }\end{array}$ & $0,732^{* *}$ & $0,696^{* *}$ & 1 & $0,784^{* *}$ & $-0,276^{* *}$ \\
\hline & Sig. (2-tailed) & 0,000 & 0,000 & & 0,000 & 0,000 \\
\hline & $\mathrm{N}$ & 2457 & 2457 & 2457 & 2457 & 2457 \\
\hline \multirow{3}{*}{$\begin{array}{l}\text { Óbitos } \\
\text { Acumulados }\end{array}$} & $\begin{array}{l}\text { Pearson } \\
\text { Correlation }\end{array}$ & $0,734^{* *}$ & $0,942^{* *}$ & $0,784^{* *}$ & 1 & $-0,349^{* *}$ \\
\hline & Sig. (2-tailed) & 0,000 & 0,000 & 0,000 & & 0,000 \\
\hline & $\mathrm{N}$ & 2457 & 2457 & 2457 & 2457 & 2457 \\
\hline
\end{tabular}




\begin{tabular}{|c|c|c|c|c|c|c|}
\hline \multirow{3}{*}{ Trend } & $\begin{array}{l}\text { Pearson } \\
\text { Correlation }\end{array}$ & $-0,386^{* *}$ & $-0,441^{* *}$ & $-0,276^{* *}$ & $-0,349^{* *}$ & 1 \\
\hline & Sig. (2-tailed) & 0,000 & 0,000 & 0,000 & 0,000 & \\
\hline & $\mathrm{N}$ & 2457 & 2457 & 2457 & 2457 & 2457 \\
\hline
\end{tabular}

Fonte: Autores.

A partir de maio de 2020, observou-se um aumento significativo do número de casos acumulados e óbitos, como pode ser visualizado no Gráfico 3.

Gráfico 3 - Evolução de casos e óbitos de COVID-19 no período abr-junho/2020, Brasil.

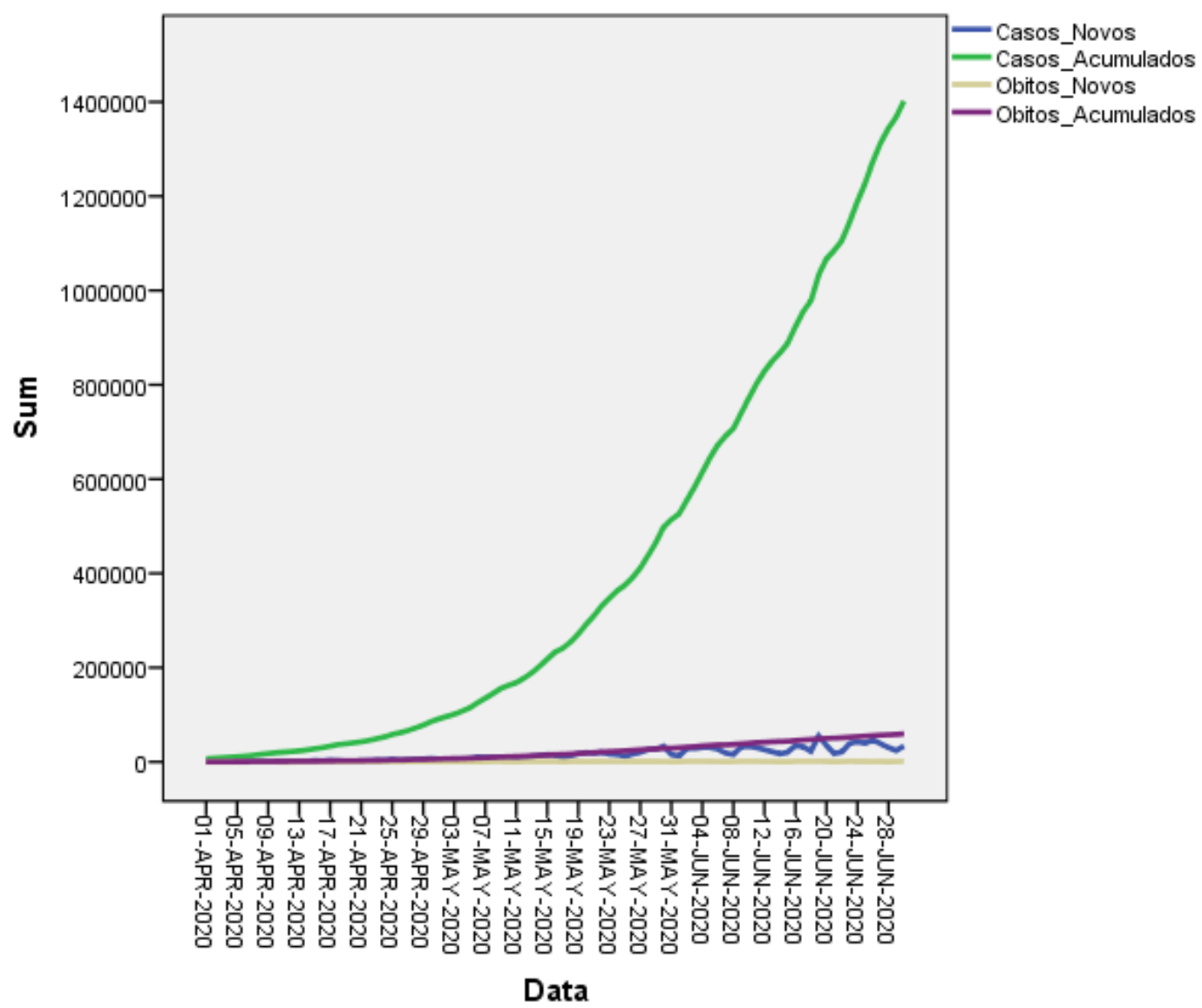

Fonte: SPSS (2020).

A média brasileira de casos acumulados atingiu o quantitativo de $15.111,80$, dentro do período estudado. O número médio de casos acumulados mostrou-se maior em São Paulo $(88.306,84)$, ao passo que a menor média de casos acumulados foi registrada em Mato Grosso do Sul, com 1.645,27. Ressalta-se que os estados se encontram em regiões geográficas distintas, Sudeste e Centro-Oeste, respectivamente. Os dados, apresentados no Quadro 1, mostram que, em determinados parâmetros epidemiológicos, UFs geograficamente distantes apresentam comportamento semelhante, o que era esperado entre UFs da mesma região geográfica. 
Quadro 1 - Casos e óbitos de COVID-19 nas Unidades Federativas e Distrito Federal brasileiro no período abr-junho/2020.

\begin{tabular}{|c|c|c|c|c|}
\hline Estados Brasileiros (UFs) & Casos novos & Casos acumulados & $\begin{array}{l}\text { Óbitos } \\
\text { novos }\end{array}$ & $\begin{array}{c}\text { Óbitos } \\
\text { acumulados }\end{array}$ \\
\hline Mato Grosso do Sul & $87^{\mathrm{G}}$ & $1645,27^{\mathrm{H}}$ & $0,82^{\mathrm{G}}$ & $19,12^{\mathrm{F}}$ \\
\hline Tocantins & $118,16^{\mathrm{G}}$ & $2978,78^{\mathrm{H}}$ & $2,2^{\mathrm{FG}}$ & $57,76^{\mathrm{F}}$ \\
\hline Acre & $145,18^{\mathrm{G}}$ & $4233,75^{\mathrm{H}}$ & $4,01^{\mathrm{EFG}}$ & $113,07^{\mathrm{F}}$ \\
\hline Roraima & $160,71^{\mathrm{G}}$ & $3307,48^{\mathrm{H}}$ & $3,11^{\mathrm{FG}}$ & $87^{\mathrm{F}}$ \\
\hline Mato Grosso & $171,55^{\mathrm{G}}$ & $2893,98^{\mathrm{H}}$ & $6,52^{\mathrm{EFG}}$ & $100,9^{\mathrm{F}}$ \\
\hline Piauí & 224,22 FG & $4683,23 \mathrm{GH}$ & $7,24^{\mathrm{EFG}}$ & $157,51^{\mathrm{F}}$ \\
\hline Rondônia & $233,44^{\mathrm{FG}}$ & $5018,75^{\mathrm{GH}}$ & $5,68^{\mathrm{EFG}}$ & $140,45^{\mathrm{F}}$ \\
\hline Paraná & $249,43^{\mathrm{FG}}$ & $5005^{\mathrm{GH}}$ & $7,1^{\mathrm{EFG}}$ & $181,12^{\mathrm{F}}$ \\
\hline Goiás & 267,21 FG & $4694,29 \mathrm{GH}$ & $5,2^{\mathrm{EFG}}$ & $115,73^{\mathrm{F}}$ \\
\hline Sergipe & 279,03 FG & $6225,01 \mathrm{GH}$ & $7,43^{\mathrm{EFG}}$ & $148,64^{\mathrm{F}}$ \\
\hline Santa Catarina & $287,20^{\mathrm{FG}}$ & $7208,14^{\mathrm{GH}}$ & $3,73^{\mathrm{FG}}$ & $111,02^{\mathrm{F}}$ \\
\hline Rio Grande do Sul & 293,04 FG & $7337,31^{\mathrm{GH}}$ & $6,7 \mathrm{EFG}$ & $182,69^{\mathrm{F}}$ \\
\hline Amapá & 312,99 FG & $7859,87 \mathrm{GH}$ & $4,58^{\mathrm{EFG}}$ & $151,74^{\mathrm{F}}$ \\
\hline Rio Grande do Norte & 331,92 EFG & $6619,76^{\mathrm{GH}}$ & $11,35^{\mathrm{EFG}}$ & $260,19^{\mathrm{F}}$ \\
\hline Alagoas & 394,99 DEFG & $9215,2^{\mathrm{GH}}$ & $11,55^{\mathrm{EFG}}$ & $330,96^{\mathrm{F}}$ \\
\hline Minas Gerais & 491,49 CDEFG & $10019,25^{\mathrm{GH}}$ & $10,58^{\mathrm{EFG}}$ & $250,97^{\mathrm{F}}$ \\
\hline Espírito Santo & $514,38^{\mathrm{CDEFG}}$ & $12378,99^{\mathrm{FGH}}$ & $18,11^{\mathrm{DEFG}}$ & $485,27^{\mathrm{F}}$ \\
\hline Paraíba & $515,82^{\mathrm{CDEFG}}$ & $11801,63^{\mathrm{FGH}}$ & $10,74^{\mathrm{EFG}}$ & $291,93^{\mathrm{F}}$ \\
\hline Distrito Federal & 537,21 CDEFG & $10668,34^{\text {FGH }}$ & $6,42^{\mathrm{EFG}}$ & $147,01^{\mathrm{F}}$ \\
\hline Pernambuco & $645,84 \mathrm{CDEF}$ & 22778,14 DEF & $53^{\mathrm{C}}$ & $1877,97 \mathrm{CD}$ \\
\hline Amazonas & 776,35 BCDE & $26660,26^{\mathrm{BCDE}}$ & $30,99^{\mathrm{D}}$ & $1316,51^{\mathrm{DE}}$ \\
\hline Bahia & 803,23 BCD & $17070,66^{\mathrm{EFG}}$ & $20,34^{\mathrm{DEF}}$ & $520,57^{\mathrm{F}}$ \\
\hline Maranhão & $883,74^{\mathrm{BC}}$ & $25129,87 \mathrm{CDE}$ & $22,49 \mathrm{C}^{\mathrm{DE}}$ & $701,79^{\mathrm{EF}}$ \\
\hline Pará & $1.133,78^{\mathrm{B}}$ & 29600,01 BCD & $54,07^{\mathrm{C}}$ & $1856,58^{\mathrm{CD}}$ \\
\hline Ceará & $1.190,21^{\mathrm{B}}$ & $36428,98 \mathrm{BC}$ & $67,46^{\mathrm{C}}$ & $2255,79^{\mathrm{C}}$ \\
\hline Rio de Janeiro & $1.229,70^{\mathrm{B}}$ & $38249,92^{\text {В }}$ & $110,52^{\mathrm{B}}$ & $3678,91^{\text {B }}$ \\
\hline São Paulo & $3066,38^{A}$ & $88306,84^{\mathrm{A}}$ & $160,74^{\mathrm{A}}$ & $5639,63^{A}$ \\
\hline
\end{tabular}

Fonte: Autores.

A média brasileira de óbitos acumulados atingiu o valor de 784,47, dentro do período estudado. O número médio de óbitos acumulados mostrou-se maior em São Paulo $(5.639,63)$, ao passo que a menor média de óbitos acumulados registrada ocorreu em Mato Grosso do Sul $(19,12)$. 
Considerando os dados, firmamos que existe uma forte relação negativa entre o acesso ao conteúdo na Internet e o número de casos confirmados e óbitos por coronavírus em todas as UFs brasileiras, como pode ser visualizado no Gráfico 4 .

Com base nos resultados, observou-se alta significância estatística para as correlações negativas entre os dados do GT e os parâmetros epidemiológicos da COVID-19 para todas as UFs e todas as categorias aplicáveis, como pode ser visualizado na Tabela 2.

Gráfico 4 - Correlação de Pearson mensal entre óbitos por COVID-19 e RSV no período abr-junho/2020, Brasil.

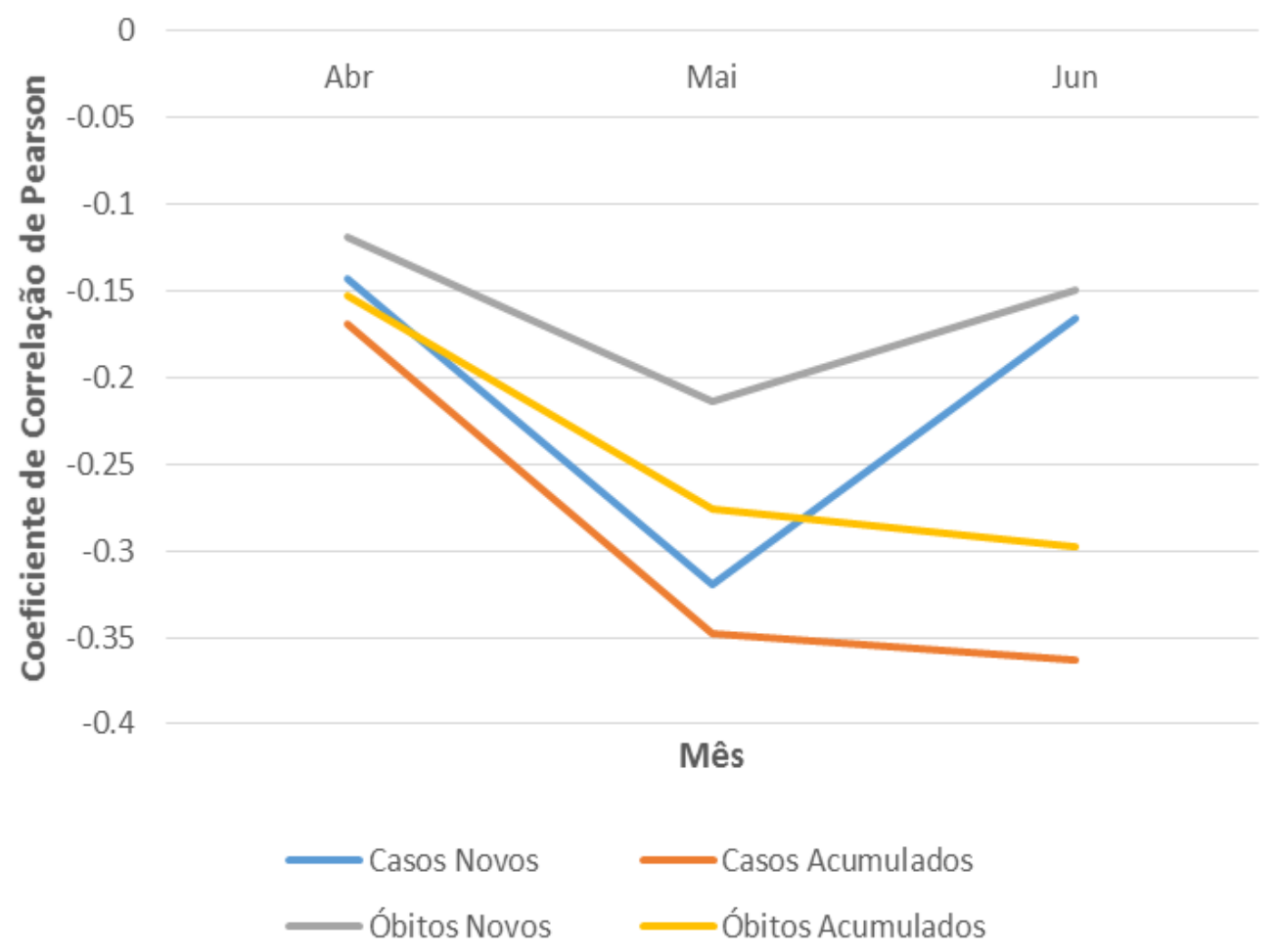

Fonte: Autores. 
Research, Society and Development, v. 10, n. 9, e1010917817, 2021

(CC BY 4.0) | ISSN 2525-3409 | DOI: http://dx.doi.org/10.33448/rsd-v10i9.17871

Tabela 2 - Correlação entre casos e óbitos de COVID-19 e RSV no período abr-junho/2020, Brasil.

\begin{tabular}{|c|c|c|c|c|c|}
\hline Região & Estado & Casos Novos & Casos Acumulados & Óbitos Novos & Óbitos Acumulados \\
\hline \multirow{7}{*}{$\begin{array}{l}\text { Região } \\
\text { Norte }\end{array}$} & Acre & $-0,696$ & $-0,822$ & $-0,673$ & $-0,817$ \\
\hline & Amapá & $-0,538$ & $-0,767$ & $-0,625$ & $-0,851$ \\
\hline & Amazonas & $-0,632$ & $-0,779$ & $-0,391$ & $-0,852$ \\
\hline & Pará & $-0,764$ & $-0,786$ & $-0,557$ & $-0,858$ \\
\hline & Rondônia & $-0,674$ & $-0,722$ & $-0,683$ & $-0,759$ \\
\hline & Roraima & $-0,422$ & $-0,677$ & $-0,447$ & $-0,735$ \\
\hline & Tocantins & $-0,822$ & $-0,829$ & $-0,645$ & $-0,828$ \\
\hline \multirow{9}{*}{$\begin{array}{l}\text { Região } \\
\text { Nordeste }\end{array}$} & Alagoas & $-0,802$ & $-0,769$ & $-0,837$ & $-0,835$ \\
\hline & Bahia & $-0,518$ & $-0,717$ & $-0,793$ & $-0,756$ \\
\hline & Ceará & $-0,568$ & $-0,817$ & $-0,603$ & $-0,842$ \\
\hline & Maranhão & $-0,768$ & $-0,822$ & $-0,872$ & $-0,871$ \\
\hline & Paraíba & $-0,69$ & $-0,79$ & $-0,742$ & $-0,844$ \\
\hline & Pernambuco & $-0,651$ & $-0,859$ & $-0,681$ & $-0,856$ \\
\hline & Piauí & $-0,7$ & $-0,716$ & $-0,718$ & $-0,713$ \\
\hline & $\begin{array}{l}\text { Rio Grande do } \\
\text { Norte }\end{array}$ & $-0,385$ & $-0,714$ & $-0,578$ & $-0,74$ \\
\hline & Sergipe & $-0,662$ & $-0,728$ & $-0,72$ & $-0,691$ \\
\hline \multirow{4}{*}{$\begin{array}{l}\text { Centro- } \\
\text { Oeste }\end{array}$} & Distrito Federal & $-0,635$ & $-0,638$ & $-0,602$ & $-0,664$ \\
\hline & Goiás & $-0,526$ & $-0,588$ & $-0,495$ & $-0,684$ \\
\hline & $\begin{array}{l}\text { Mato Grosso do } \\
\text { Sul }\end{array}$ & $-0,594$ & $-0,602$ & $-0,373$ & $-0,669$ \\
\hline & Mato Grosso & $-0,532$ & $-0,541$ & $-0,475$ & $-0,505$ \\
\hline \multirow{4}{*}{ Sudeste } & Espírito Santo & $-0,725$ & $-0,747$ & $-0,772$ & $-0,775$ \\
\hline & Minas Gerais & $-0,484$ & $-0,66$ & $-0,603$ & $-0,713$ \\
\hline & Rio de Janeiro & $-0,607$ & $-0,816$ & $-0,684$ & $-0,846$ \\
\hline & São Paulo & $-0,626$ & $-0,784$ & $-0,631$ & $-0,847$ \\
\hline \multirow{3}{*}{ Sul } & Paraná & $-0,54$ & $-0,601$ & $-0,485$ & $-0,686$ \\
\hline & $\begin{array}{l}\text { Rio Grande do } \\
\text { Sul }\end{array}$ & $-0,596$ & $-0,732$ & $-0,574$ & $-0,786$ \\
\hline & Santa Catarina & $-0,587$ & $-0,757$ & $-0,507$ & $-0,796$ \\
\hline
\end{tabular}

Fonte: Autores.

\section{Discussão}

O Brasil continua registrando aumento de casos de COVID-19, configurando-se atualmente como um dos epicentros da pandemia. O país contabilizou, em julho de 2021, mais de 18 milhões de casos. Na América do Sul, Equador, Bolívia, Peru, 
Guiana e Colômbia superam o Brasil em taxa de mortalidade (OMS, 2021; Carvalho, Bochiero \& Marson, 2021; Marinho, Cordeiro, Coelho \& Brandão, 2021).

De acordo com os dados do nosso estudo, 19 das 27 unidades federativas apresentavam crescimento lento de novos casos e óbitos, de modo que poderiam ter sido adotadas medidas preventivas para controlar o avanço da doença no Brasil.

Lobo, Cardoso-dos-Santos, Rocha, Pinheiro, Bremm, Macário et al. (2020), analisaram a média diária de casos e determinou que as regiões com maiores valores de variação percentual média diária foram as regiões Norte, Nordeste e Sudeste. Em relação às UFs, foi evidenciado que cada uma se encontra em um estágio diferente da pandemia, mas todas apresentam tendência de aumento no número de casos de COVID-19. Consideramos que os diferentes estágios da pandemia nos estados brasileiros e no Distrito Federal possuem relação com a extensão territorial, cultural e socioeconômica.

Nossos dados também mostram que, em determinados parâmetros epidemiológicos, unidades federativas geograficamente distantes apresentam comportamento semelhante, o que era esperado entre UFs da mesma região geográfica. Isto mostra que o Brasil apresenta diversos clusters e com comportamentos distintos, o que dificulta a adoção de medidas sanitárias nacionais, de modo que devem ser implementadas determinando medidas unificadas, mas considerando as características locais.

Estudos epidemiológicos baseados em testes estatísticos identificaram clusters de alto risco para COVID-19 no Brasil nas regiões costeiras, onde estão localizadas a maioria das capitais das UFs, com tendência de dispersão para a região metropolitana e para as cidades do interior. Esses achados alertavam para o colapso dos sistemas públicos de saúde, pois a maioria das cidades do interior, mesmo possuindo um sistema de saúde pública implantado, não possui estrutura hospitalar para atender aos casos de complexidade elevada da COVID-19 (Gomes, Andrade, Ribeiro, Peixoto, Lima, Duque et al. 2020; Almeida, Pronunciate, Grotto, Pugliesi, Guimarães, Vilches et al. 2020; Amaral, Santos, Figueredo, Ferreira, Silva, Santos, et al., 2020; Dias, Pamplona, Rodrigues, Vogado, Silva-Júnior, Barreto et al., 2020).

A tendência de aumento da COVID-19 na região Nordeste sofre impacto e impacta a Europa. Essa região brasileira é a mais próxima do continente europeu e possui tráfego aéreo nacional e internacional intenso, o que pode ajudar a explicar a maior incidência de COVID-19 na região Nordeste do Brasil. A estimativa de risco relativo da introdução da COVID-19 em cidades brasileiras por viajante internacional mostra que mais da metade dos casos seriam de viajantes infectados na Itália, país com alta taxa de mortalidade, seguidos de viajantes infectados na China, país com alta taxa de transmissão, e França (Gomes, Andrade, Ribeiro, Peixoto, Lima, Duque et al., 2020; Almeida, Pronunciate, Grotto, Pugliesi, Guimarães, Vilches et al., 2020; Cândido, Watts, Abade, Kraemer, Pybus, Croda et al, 2020).

O estado de São Paulo, localizado na região Sudeste do Brasil, é a UF mais rica e populosa do país, com aproximadamente 45 milhões de habitantes, o que representa aproximadamente $21,7 \%$ da população brasileira, e é o que apresenta o maior número de casos desde o início da pandemia. Muito se deve justamente a densidade populacional elevada (Lobo, Cardoso-dos-Santos, Rocha, Pinheiro, Bremm, Macário, et al., 2020; Almeida, Pronunciate, Grotto, Pugliesi, Guimarães, Vilches et al., 2020; Serdan, Mais, Gorjao, Pithon-Curi, Curi \& Hirabara, 2020; Neiva, Carvalho, Costa-Filho, Barbosa-Junior, Bernardi, Sanches et al, 2020; Dias, Pamplona, Rodrigues, Vogado, Silva-Júnior, Barreto et al., 2020).

Até o dia 7 de março, o Ministério da Saúde do Brasil notificou 19 casos, dos quais 11 eram de pessoas vindas da Itália. Destes, três eram pessoas vindas da Itália com passagem por outros países, como Alemanha, Espanha e Austrália; três vinham de outros países, como Estados Unidos, Reino Unido e Irã; e dois casos de transmissão local associados ao primeiro caso, que ocorreram nas cidades de São Paulo e Rio de Janeiro. Em 13 de março de 2020 já eram contabilizados 98 casos. Após 63 dias, em 12 de maio de 2020, 177,589 casos haviam sido confirmados, a maioria no estado de São Paulo (Almeida, Pronunciate, Grotto, Pugliesi, Guimarães, Vilches et al., 2020; Serdan, Mais, Gorjao, Pithon-Curi, Curi \& Hirabara, 2020; Neiva, Carvalho, Costa-Filho, Barbosa-Junior, Bernardi, Sanches et al, 2020). 
Desde a confirmação da pandemia, o Brasil teve dois meses até notificar o primeiro caso de COVID-19 em seu território. Nesse ínterim, o governo brasileiro teve tempo de adquirir informações e conhecimento para entender melhor a evolução da doença, bem como a oportunidade de adotar medidas exitosas de outros países. Entretanto, cinco meses após o primeiro caso confirmado, tornou-se o segundo país com o maior número de casos, apresentando curva acentuada em maio de 2020 (Neiva, Carvalho, Costa-Filho, Barbosa-Junior, Bernardi, Sanches et al, 2020; Cimerman, Chebabo, Cunha \& Rodrígez-Morales, 2020).

Entre as UFs, São Paulo, Rio de Janeiro, Ceará, Pernambuco, Bahia e Pará apresentam os maiores números de casos acumulados e óbitos. No final de maio de 2020, eles registraram cerca de 880 mil casos, isto é, $47 \%$ do total de casos brasileiros. Em julho de 2020, representaram 75\% do total de mortes brasileiras. Em 2021, São Paulo, Rio de Janeiro, Ceará e Pernambuco ainda apresentam as maiores taxas de letalidade do Brasil. São Paulo lidera em número de casos desde o início, com aproximadamente $173 \%$ a mais de casos do que o Ceará (Neiva, Carvalho, Costa-Filho, Barbosa-Junior, Bernardi, Sanches et al, 2020; Marinho, Cordeiro, Coelho \& Brandão, 2021).

As UFs brasileiras possuem autonomia administrativa, de modo que diversas delas adotaram protocolos de segurança da OMS, como medidas de distanciamento social, testagem da população, isolamento de casos positivos, uso obrigatório de máscara pela população em geral e protocolos de segurança de saúde para atividades essenciais e não essenciais. Assim, em conjunto, reduziram a velocidade de dispersão do vírus, mas foram insuficientes para interromper a transmissão. Vale destacar que parte da população se mostrou resistente a adotar as medidas preventivas (Almeida, Pronunciate, Grotto, Pugliesi, Guimarães, Vilches et al., 2020).

Somente uma pequena porcentagem de brasileiros foi testada, ou por escassez de insumos, ou pelo alto custo dos exames. A reduzida testagem associada com a subnotificação torna a situação ainda mais preocupante, pois os países com as taxas mais altas de testes são considerados casos de sucesso na pandemia (Neiva, Carvalho, Costa-Filho, Barbosa-Junior, Bernardi, Sanches et al, 2020; Almeida, Pronunciate, Grotto, Pugliesi, Guimarães, Vilches et al., 2020; Ferreira, Almeida, Mattos \& Oliveira, 2020; Sousa, Torres, Moura, Silva, Araújo, Sousa et al., 2020; Carvalho, Bochiero \& Marson, 2021; Marinho, Cordeiro, Coelho \& Brandão, 2021).

Wang, Pan, Zhang, Fan, Han, Zhao et al. (2020) destacam dois pontos para o controle do avanço da COVID-19: testar pacientes sem histórico de exposição e identificar pacientes infectados assintomáticos, considerando que a gravidade da doença pode ser reduzida com o diagnóstico e o atendimento médico-hospitalar precoce.

Recentemente, muitas cidades brasileiras reduziram suas restrições em relação a exigência de quarentena em resposta à redução da incidência de novos casos. Tudo sugere que o Brasil reduziu as restrições prematuramente, considerando apenas os números de casos, sem analisar profundamente o sistema de atendimento de saúde local e, por esse motivo, pode enfrentar graves consequências em um futuro próximo. Na saúde pública, a prevenção é sempre a opção menos onerosa, principalmente neste caso, em que a resposta de um indivíduo à infecção pode ser a morte (Lobo, Cardoso-dos-Santos, Rocha, Pinheiro, Bremm, Macário, et al., 2020; Almeida, Pronunciate, Grotto, Pugliesi, Guimarães, Vilches et al., 2020; Neiva, Carvalho, Costa-Filho, Barbosa-Junior, Bernardi, Sanches et al, 2020; Dias, Pamplona, Rodrigues, Vogado, Silva-Júnior, Barreto et al., 2020; Marinho, Cordeiro, Coelho \& Brandão, 2021).

A adoção da quarentena comunitária e do distanciamento social são medidas preventivas que podem trazer impactos econômicos e sociais negativos, mas quando efetivamente implementadas retardam significativamente o surto de COVID-19. Essas medidas requerem comprometimento do governo e da população, para a sua adequada adoção e para gerenciar as suas consequências (Zhu, Lib, Wuc, Xud, Lia, Yanga et al., 2020; Ferreira, Almeida, Mattos \& Oliveira, 2020; Silva \& Pimentel, 2021).

Os números de casos e de mortes no início da pandemia, no Brasil, ainda não eram tão alarmantes, dando à população uma falsa sensação de segurança, o que pode ter influenciado na não adoção das medidas preventivas, mas, ainda hoje, as medidas 
preventivas são negligenciadas por muitos brasileiros, aumentando a disseminação do vírus (Almeida, Pronunciate, Grotto, Pugliesi, Guimarães, Vilches et al., 2020; Neiva, Carvalho, Costa-Filho, Barbosa-Junior, Bernardi, Sanches et al, 2020; Marinho, Cordeiro, Coelho \& Brandão, 2021).

No dia 13 de março de 2020 foi notificado o primeiro caso na região Norte do Brasil, no Amazonas. Dados estimativos mostravam que antes do final de abril de 2020 o Amazonas teria mais de 50.000 pessoas infectadas caso não fosse adotada nenhuma medida de distanciamento social. Se fosse adotada a medida preventiva, e cerca de $20 \%$ da população ficasse em quarentena, os números cairiam para menos de 50.000. Com a adoção da quarentena por 50\% da população, os números cairiam e apenas em maio atingiriam 50.000. Além disso, com a adoção do lockdown, esse valor só seria alcançado em junho, não comprometendo o sistema de saúde (Bitar \& Steinmetz, 2020).

Em abril de 2020, os estados da região Norte, Acre, Amapá, Amazonas e Roraima, apresentaram alta transmissão comunitária. Isto resultou em um novo foco no território brasileiro, que culminou em um colapso no sistema de saúde em cidades com estrutura médico-hospitalar deficiente, por apresentar baixo percentual de leitos de UTI e alta necessidade de equipamentos médicos, como ventiladores mecânicos (Bastos, Passos, Silva \& Santos, 2016; Neiva, Carvalho, Costa-Filho, Barbosa-Junior, Bernardi, Sanches et al, 2020).

Nossos dados corroboram Marinho, Cordeiro, Coelho \& Brandão (2021), quando dizem que o aumento, a redução e a tendência de aumento de números de casos e óbitos variam de forma diferente em cada UF do Brasil.

Mavragani (2020) identificou correlações estatisticamente significativas entre o interesse ao acesso à informação online e os casos e mortes por COVID-19, afirmando que os dados de pesquisa on-line estão altamente correlacionados com casos e mortes por COVID-19 e que, dessa forma, os dados do GT podem ajudar no rastreamento da transmissão. O autor afirma que em regiões pouco afetadas pela COVID-19 há mais buscas na Internet.

Os nossos dados mostram que o Brasil não segue essa tendência, pois as regiões pouco afetadas pela COVID19 apresentaram RSV semelhante às severamente afetadas, o que, aparentemente, impossibilita a adoção da que a infodemiologia pelos gestores para identificar onde ocorrerá aumento nos casos de COVID-19 e adotar, com antecedência, medidas para minimizar a propagação da doença. Entretanto não descartamos a possibilidade do uso da pesquisa infodemiológica associada aos dados dos sistemas de saúde nacionais. Caso encontrem uma correlação positiva, que façam uso da ferramenta em benefício da população. Já que, segundo Effenberger, Kronbichler, Shin, Mayer, Tilg \& Perco (2020) e Tana (2019), os padrões e variações rítmicos claros e robustos dos RSVs do GT podem ser identificáveis e servir para gerenciamento de ações em saúde em nível local, permitindo acompanhar variações temporais dos comportamentos, fornecendo uma alternativa para prever dados comportamentais relacionados à saúde.

Quando as taxas de doença e a atividade de pesquisa na Internet concordam no mesmo período, essa correlação reforça o uso da infodemiologia no monitoramento de doenças em uma população. Dados gerados por GT, quando correlacionados com banco de dados governamentais, podem ser explorados para complementar a vigilância epidemiológica tradicional para doenças infectocontagiosoas, incluindo a COVID-19 (Bragazzi, Bacigaluppi, Robba, Siri, Canepa \& Brigo, 2016; Ling \& Lee, 2016; Strzelecki \& Rizun, 2020). Os dados brasileiros aqui apresentados, entretanto, se comportaram de forma diferente, sendo observado um comportamento inverso ao sugerido pelos autores.

Além do mais, os gestores devem ter atenção, pois, em alguns casos, o interesse em pesquisar na Internet sobre um tema de saúde pode começar a diminuir, independentemente dos novos casos não diagnosticados, pois o crescimento do interesse pode desacelerar à medida que aumenta a conscientização (Strzelecki \& Rizun, 2020; Mavragani, 2020). No caso específico do Brasil, devem ficar atentos à subnotificação, que pode comprometer uma nova análise da situação (Sousa, Torres, Moura, Silva, Araújo, Sousa et al., 2020). 
As questões locais sobre o controle da epidemia ainda são um desafio para os estudos brasileiros. Deve-se levar em consideração que o Brasil é um país bastante heterogêneo e com grande desigualdade social, pois cerca de 11,4 milhões de pessoas vivem em situação de risco socioeconômico, o que faz com que a pandemia afete o sistema público de saúde e a população de forma não uniforme. Portanto, é essencial explorar diferentes ferramentas de monitoramento para identificar os padrões epidemioógicos, e com eles adotar medidas locais o mais rápido possível, avaliando os resultados das ações de maneira periódica (Dias, Pamplona, Rodrigues, Vogado, Silva-Júnior, Barreto et al., 2020; Almeida, Pronunciate, Grotto, Pugliesi, Guimarães, Vilches et al., 2020; Neiva, Carvalho, Costa-Filho, Barbosa-Junior, Bernardi, Sanches et al, 2020; Barros, Romão, Laudares, Morares \& Gomes, 2020; Cardoso, Alberto, Maués, Silva, Abreu, Coelho et al., 2021).

Concordamos com Mavragani, Ochoa \& Tsagarakis (2018) quando dizem que a análise do comportamento on-line por região pode, em um futuro próximo, ajudar a acompanhar a transmissão de prevalência de doenças, bem como auxiliar na implementação de intervenções e campanhas de saúde direcionadas durante períodos em que se prevê aumento do número de casos de determinada doença, proporcionando alocação econômica de recursos e uma relação custo-benefício focada na saúde da população.

O presente estudo traz resultados inéditos e relevantes, mas reconhecemos quatro limitações: a primeira se refere à falta de padronização metodológica; a segunda deve-se ao fato de que a pandemia ainda se encontra em progressão; a terceira se refere à forma como o GT disponibiliza dos dados; e a quarta se refere ao usuário que realizou a busca na Internet.

Em relação à padronização metodológica, Nuti, Wayda, Ranasinghe, Wang, Dreyer, Chen et al. (2014), também compartilham dessa opinião, mas Mavragani, Ochoa \& Tsagarakis (2018) defendem a infodemiologia como uma promissora ferramenta de pesquisa no campo do comportamento coletivo.

Em relação ao andamento da pandemia, os resultados estão sujeitos a alterações à medida que avançamos, mas, sem dúvida, a presente pesquisa traz um recorte temporal valioso da realidade estudada, sendo relevante para a data relatada.

Em relação aos dados do GT, este é o único e atual mecanismo de pesquisa que oferece uma análise de dados em tempo real, acessível ao público e que permite a publicação de pesquisas semelhantes em periódicos de grande valor cientifico.

Em relação ao usuário que realizou o acesso, é impossível saber em qual circunstância se realizou, isto é, se foi por pessoas saudáveis, pacientes, cuidadores ou profissionais de saúde, bem como não é possível saber qual a finalidade das buscas. Esta é uma limitação clássica de conduzir um estudo observacional em que seria impossível discernir uma relação de causa e efeito.

\section{Conclusão}

A pesquisa utilizando o comportamento da população brasileira em busca de informações no buscador Google não permitiu evidenciar uma correlação positiva entre os dados epidemiológicos, o número de infectados e os óbitos por COVID-19 no Brasil no período estudado, mas este breve artigo é um ponto de partida para uma análise mais aprofundada, no campo da infodemiologia, da pandemia da doença do novo coronavírus no Brasil.

Nossos dados podem servir para informar aos gestores que atualmente estão disponíveis diversas ferramentas para uma adequada gestão em saúde. Esperamos que as informações aqui publicadas possam fornecer informações valiosas para agências de saúde pública, brasileira e internacionais, em meio a uma pandemia que trouxe uma crise global de saúde pública. Esperamos, também, que alguma medida baseada em conhecimento científico seja adotada para controlar os danos causados na população brasileira.

A metodologia infoepidemiológica precisa ser divulgada para que os pesquisadores possam compreender melhor as tendências de busca por informações de saúde na Internet e na pesquisa em saúde, propondo o uso de dados de comportamento on-line para ampliar o entendimento das informações em saúde. 
Consideramos que a associação dessas ferramentas pode ser usada como uma ferramenta de gestão para associar e prever agravos em saúde no território brasileiro. Nesse sentido, independente da ferramenta a ser adotada, é necessário que estudos futuros continuem acompanhando os dados de comportamento social e o número de infectados e óbitos por COVID-19 a fim de verificar o comportamento atual, no Brasil e em outros países.

\section{Referências}

Almeida, G. B., Pronunciate, M., Grotto, R. M. T., Pugliesi, E. A., Guimarães, R. B., Vilches, T. N., et al. (2020). Two hundred days of COVID-19 in São Paulo State, Brazil. Epidemiol Infect, 148, 1-4.

Amaral, L. S., Santos, A. L. P., Figueredo, M. P. S., Ferreira, D. S. A., Silva, J. E., Santos, H. C. T., et al. (2020). Interiorização do Covid-19: Uma análise da evolução dos casos/10 mil habitantes em municípios da Microrregião de Garanhuns no Estado de Pernambuco, através de modelos de Regressão não linear. Research, Society and Development, 9 (9), 1-19.

Barros, A. J., Romão, E. M., Laudares, S., Morares, R. A. \& Gomes, G. G. (2020). The interdisciplinarity of geography and geoprocessing in the mapping of Covid 19 cases in the municipality of João Monlevade- Minas Gerais. Research, Society and Development, 9 (10), 1-18.

Bastos, I. L. G., Passos, A. V., Silva, J. A. \& Santos, R. A. (2016). Infecção hospitalar no Centro Cirúrgico: principais agentes causadores, fatores de riscos e medidas de prevenção. Revista Madre Ciência Saúde, 1, 39-53.

Bitar, S. \& Steinmetz, W. A. (2020). Scenarios for the Spread of COVID-19 in Manaus, Northern Brazil. An Acad Bras Cienc, 92 , 1-12.

Bragazzi, N. L., Bacigaluppi, S., Robba, C., Siri, A., Canepa, G. \& Brigo, F. (2016). Infodemiological data of West-Nile vírus disease in Italy in the study period 2004-2015. Data in Brief, 9, 839-845.

Cândido, D. S., Watts, A., Abade, L., Kraemer, M. U. G., Pybus, O. G., Croda, J., et al. (2020). Routes for COVID-19 importation in Brazil. J Travel Med, 27, $1-3$.

Cardoso RF, Alberto DS, Maués SCC; Silva SEM, Abreu AC, Coelho JSM, et al. (2021). COVID-19: An epidemiological challenge. Research, Society and Development, $10(7), 1-9$.

Carvalho, T. A., Bochiero, M. N. \& Marson, F. A. L. (2021). COVID-19 in Brazil: 150,000 deaths and the Brazilian underreporting. Diagnostic Microbiology and Infectious Disease, 99, 1-3.

Chen, N., Zhou, M., Dong, X., Qu, J., Gong, F., Han, Y., et al. (2020). Epidemiological and clinical characteristics of 99 cases of 2019 novel coronavirus pneumonia in Wuhan, China: a descriptive study. The Lancet, 395, 507-513.

Cimerman, S., Chebabo, A., Cunha, C. A. \& Rodrígez-Morales, A. J., (2020). Deep impact of COVID-19 in the healthcare of Latin America: the case of Brazil. Braz J Infect Dis, 4, 93-95.

Dias, G. N., Pamplona, V. M. S., Rodrigues, A. E., Vogado, G. E. R., Silva-Júnior, W. L., Barreto, W. D. L., et al. (2020). Mathematical and statistical analysis of disease COVID-19 and implications for future projections. Research, Society and Development, 9 (10), 1-23.

Effenberger, M., Kronbichler, A., Shin, J., Mayer, G., Tilg, H. \& Perco, P. (2020). Association of the COVID-19 pandemic with Internet Search Volumes: A Google TrendsTM Analysis. International Journal of Infectious Diseases, 95, 192-197.

Eysenbach G. (2009). Infodemiology and Infoveillance: Framework for an Emerging Set of Public Health Informatics Methods to Analyze Search, Communication and Publication Behavior on the Internet. J Med Internet Res, 11, 1-10.

Ferreira, C. M., Almeida, D. D. C., Mattos, M. L. A. D. \& Oliveira, T. K. B. (2020). COVID 19: Relação do padrão epidemiológico da COVID-19 entre China e Itália. Research, Society and Development, 9 (7), 1-12.

Gomes, D. S., Andrade, L. A., Ribeiro, C. J. N., Peixoto, M. V. S., Lima, S. V. M. A., Duque, A. M., et al. (2020). Risk clusters of COVID-19 transmission in northeastern Brazil: prospective space-time modelling. Epidemiol Infect, 148, 1-8.

Hosseini, S. S., Asemi, A. \& Shabani, A. (2019). Infodemiology in Public Health Infoveillance: An Inevitable Necessity in 21st Century. Knowledge and Information Management, 5, 49-62.

Huang, C., Wang, Y., Li, X., Ren, L., Zhao, J., Hu, Y., et al. (2020). Clinical features of patients infected with 2019 novel coronavírus in Wuhan, China. The Lancet, 395, 497-506.

Instituto Brasileiro de Geografia e Estatítica. (2021). Cidades Estados http:// www.ibge.gov.br/cidades-e-estados.html?view=município

Ling, R. \& Lee, J. (2016). Disease Monitoring and Health Campaign Evaluation Using Google Search Activities for HIV and AIDS, Stroke, Colorectal Cancer, and Marijuana Use in Canada: A Retrospective Observational Study. JMIR Public Health Surveill, 2, 1-15.

Lobo, A. P., Cardoso-dos-Santos, A. C., Rocha, M. S., Pinheiro, R. S., Bremm, J. M., Macário, E. M., et al. (2020). COVID-19 epidemic in Brazil: Where are we at?. Int J Infect Dis, 97, 382-385.

Marinho, P. R. D., Cordeiro, G. M., Coelho, H. F. C. \& Brandão, S. C. S. (2021). Covid-19 in Brazil: A sad scenario. Cytokine \& Growth Factor Reviews, 58, $51-54$. 
Research, Society and Development, v. 10, n. 9, e1010917817, 2021

(CC BY 4.0) | ISSN 2525-3409 | DOI: http://dx.doi.org/10.33448/rsd-v10i9.17871

Mavragani, A., Ochoa, G. \& Tsagarakis, K. P. (2018). Assessing the methods, tools, and statistical approaches in Google Trends research: systematic review. $J$ Med Internet Res, 20, 1-20.

Mavragani, A. (2020). Tracking COVID-19 in Europe: Infodemiology Approach. JMIR Public Health Surveill, 6, 1-13.

Moretti, F. A., Oliveira, V. E. \& Silva, E. M. K. (2012). Acesso a informações de saúde na internet: uma questão de saúde pública? Rev Assoc Med Bras, 58, $650-658$.

Neiva, M. B., Carvalho, I., Costa-Filho, E. S., Barbosa-Junior, F., Bernardi, F. A., Sanches, T. L. M., et al. (2020). Brazil: the emerging epicenter of COVID-19 pandemic. Rev Soc Bras Med Trop, 53, 1-8.

Nuti, S. V., Wayda, B., Ranasinghe, I., Wang, S., Dreyer, R. P., Chen, S. I., et al. (2014). The Use of Google Trends in Health Care Research: A Systematic Review. Plos One, 9, 1-49.

Organização Mundial da Saúde. (2021). Painel do Coronavirus da OMS (COVID-19) http://covid19.who.int/.

Serdan, T. D. A., Mais, L. N., Gorjao, R., Pithon-Curi, T. C., Curi, R., \& Hirabara, S. M. (2020). COVID-19 in Brazil: Historical cases, disease milestones, and estimated outbreak peak. J Travel Med, 38, 1-2.

Silva, M. N. R. M. O. \& Pimentel, A. S. G. (2021). Unveiling social isolation in the daily life of the COVID 19 pandemic. Research, Society and Development, $10(3), 1-12$.

Sousa, H. M., Torres, N. M. F., Moura, M. C. L., Silva, R. P., Araújo, V. L. L., Sousa, R. A., et al. (2020). Comparação de dados sobre óbitos por Covid-19 entre três fontes de informação, Maranhão. Research, Society and Development, 9 (9), 1-13.

Strzelecki, A. \& Rizun, M. (2020). Infodemiological Study Using Google Trends on Coronavirus Epidemic in Wuhan, China. IJOE, 16, 139-146.

Tana, J. (2019). Infodemiology Studying rhythmicity in online health information behaviour. Åbo Akademi University.

Wang, R., Pan, M., Zhang, X., Fan, X., Han, M., Zhao, F., et al. (2020). Epidemiological and clinical features of 125 Hospitalized Patients with COVID-19 in Fuyang, Anhui, China. International Journal of Infectious Diseases, 95, 421-428.

Zhu, N., Zhang, D., Wang, W., Li, X., Yang, B., Song, J., et al. (2020). A Novel Coronavirus from Patients with Pneumonia in China, 2019. The New England Journal of Medicine, 382 (8), 727-733.

Zhu, W., Lib, X., Wuc, Y., Xud, C., Lia, L., Yanga, J., et al. (2020). Community quarantine strategy against coronavirus disease 2019 in Anhui: An evaluation based on trauma center patients. International Journal of Infectious Diseases, 96, 417-421. 